\title{
Optimized Manufacturing of Industrial Hydrogel Sensors
}

\author{
Markus Windisch 1 , Klaus-Jochen Eichhorn², Jens Lienigi', Roland Schulze ${ }^{2}$ \\ ${ }^{1}$ Dresden University of Technology, Institute of Electromechanical and Electronic Design, \\ 01062 Dresden, Germany \\ markus.windisch@tu-dresden.de \\ ${ }^{2}$ Leibniz Institute of Polymer Research Dresden, Hohe Strasse 6, 01069 Dresden, Germany
}

\begin{abstract}
:
Fast industrial hydrogel sensors require thin polymer films with reproducible properties as sensing layer. The manufacturing process of such hydrogel coatings in the submicrometer range has been investigated with respect to a sufficient and reproducible sensitivity in order to create the necessary knowledge for a quality-assuring volume production. Interrelations between process parameters, the resulting hydrogel coating properties and the sensor behavior have been studied by means of batch manufacturing tests and direct measurements of film parameters. Furthermore, the manufacturing process of a sensor solution for surface technology with PVA/PAA hydrogel as sensing layer has been successfully optimized. The presented basic coating process and methods for its application-specific optimization are applicable for various industrial sensor designs with different hydrogels.
\end{abstract}

Key words: hydrogel sensor, PVA/PAA hydrogel, manufacturing process, surface technology

\section{Introduction}

Stimuli-sensitive hydrogels are swellable, crosslinked polymer networks. They respond to changes of the $\mathrm{pH}$-value or the concentration of certain kinds of ions respectively organics with well-defined, reversible shifts of their swelling degree. This chemo-mechanical transducer effect together with the wide diversity of available gels qualifies them as versatile sensing layers for a multiplicity of measurement tasks [1]. However, despite more than 1000 scientific publications in the last decade and numerous promising designs, hydrogel sensors have still not reached the commercial product level. One reason for that is the lack of well investigated, controllable and scalable manufacturing processes for thin hydrogel layers in the lower micrometer or submicrometer range. Due to the slow, diffusion driven swelling of hydrogels, only such thin films meet the requirement of industrial sensing applications for response times of less than a minute.

In order to address this shortcoming, we performed one of the first process-oriented investigations of the manufacturing of thin hydrogel layers using a sensor solution for monitoring the cleaner concentration in surface technology. The contributions of this paper are the presentation of a versatile basic coating process together with methods for its sensor-specific optimization. Our approach can be applied to a wide range of hydrogel sensors for industrial, biomedical and other applications.

\section{Sensor Principle and Motivation}

The sensor, previously presented in [2], consists of a piezoelectric thickness shear resonator (TSR, AT-cut quartz crystal) as highly sensitive transducer with a $200 \mathrm{~nm}$ thick PVA/PAA (polyvinyl alcohol / polyacrylic acid) hydrogel coating as sensing layer. These two coupled elements form an electro-mechanical vibration system. It shifts its frequency-dependent impedance if the hydrogel changes its swelling degree together with its mass and complex shear modulus. The sensor is excited with an AC voltage in a frequency range of $\pm 20 \mathrm{kHz}$ around the resonance frequency (about $10 \mathrm{MHz}$ ) and the corresponding current is measured. The subsequent preprocessing calculates from these values the electrical impedance and converts it to the frequency domain. Fig. 1 shows examples of the resulting impedance spectra, from whose changes the measured concentration is finally calculated through further data processing steps (details published in [3]).

The impedance spectrum of a TSR with a thin visco-elastic hydrogel film depends on four parameters [4]:

- thickness $d$

- density $\rho$

- storage modulus $G^{\prime}$

- loss modulus G"

While the density of swollen hydrogel varies at the utmost between $1 \mathrm{~g} / \mathrm{cm}^{3}$ (water) and 
$1.35 \mathrm{~g} / \mathrm{cm}^{3}$ (dry polymer) and can therefore be regarded as nearly constant, the other parameters strongly depend on the manufacturing process. Due to the high sensitivity of the TSR, the thickness $d$ and the complex shear modus $G^{*}=G^{\prime}+i G^{\prime \prime}$ must be kept within narrow tolerance ranges in order to assure an optimal and reproducible sensor function.

The quality criteria for the sensor is its sensitivity, which is defined as the concentration dependent frequency shift of the inflection point of the impedance spectra $S=\partial f_{\mathrm{ip}} / \partial c$ (Fig. 1 a). Other deviations, such as offsets of the frequency or the impedance, can be corrected with appropriate measurement processing algorithms and calibration procedures [3]. However, an insufficient sensitivity derogates the signal-to-noise-ratio and consequently directly limits the achievable measurement accuracy.

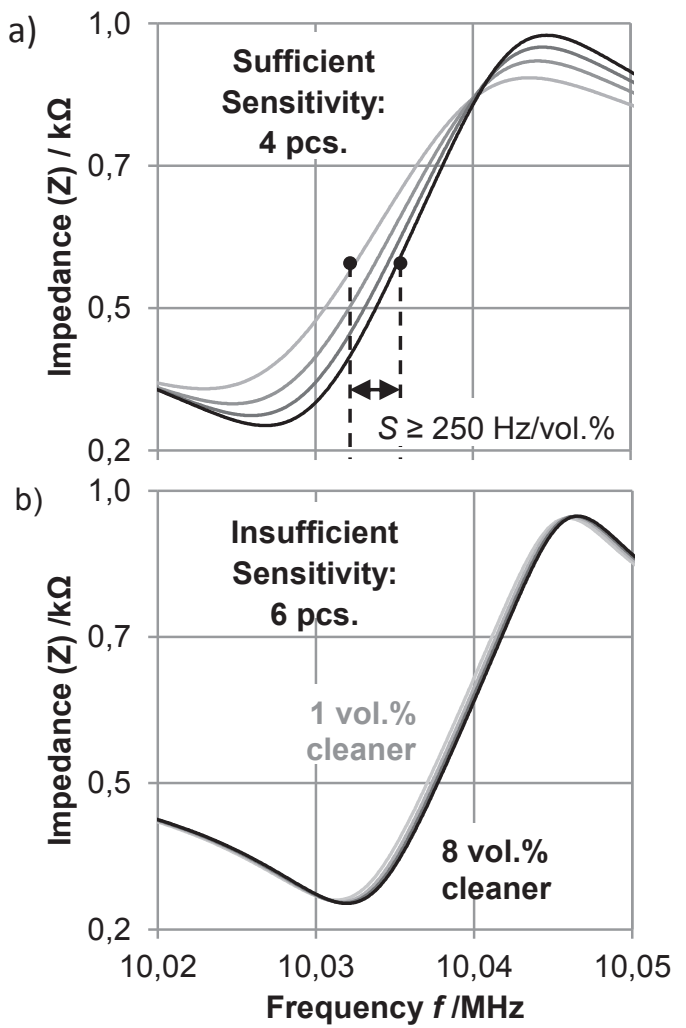

Fig. 1: Variance of sensor sensitivity in sample batch a) Good quality b) Insufficient quality

In order to investigate the manufacturability of the developed sensor, a sample batch with a volume of ten pieces was produced and characterized. Fig. 1 shows examples for the different sensor characteristics that occurred. The selected application requires at least a sensitivity of $250 \mathrm{~Hz} / \mathrm{vol} . \%$ to achieve the necessary measurement accuracy for the cleaner concentration. Using this criteria, the yield of the batch was $40 \%$, which corresponds to our experiences from previous manufacturing tests. This low yield more than doubles the effective sensor cost, which puts them out of economical range for many industrial applications, for example in surface technology.

Hence, it is necessary to investigate the interrelation between process parameters and the function determining hydrogel film properties in order to improve the yield, resulting in an optimized manufacturing technology. Furthermore, research and subsequently volume production requires suitable measurement procedures for these parameters.

\section{Manufacturing Process for Thin Hydrogel Layers}

Spin-coating was chosen as technological basis for creating thin hydrogel layers on substrates. It produces homogenous films with a controllable thickness, is well investigated and can be performed on scalable standard equipment of the semiconductor industry. Beyond these advantages, it also imposes three restrictions for the overall process and the hydrogel synthesis:

- All hydrogel components must be soluble in the same solvent.

- The viscosity of the solution must be well defined and constant.

- The cross-linking needs to take place in dry polymer, since the solvent must vaporize during the spin-coating in order to obtain uniform and stable films.

One consequence of these restrictions is that straight chemical cross-linking is not compatible with spin-coating. In several pretests with various hydrogels even long gelation times of up to 40 min led to a constant drift of the solution viscosity, which prevented the batch coating of films with a constant thickness. Applicable are generally radiation chemical, photochemical or thermal cross-linking methods of which the latter one was chosen for PVA/PAA.

Based on these preliminary considerations the following manufacturing process was used for the subsequent studies:

- Deposition of mercaptoundecanoic acid from a $1 \mathrm{mM}$ ethanol solution as adhesion promoter between the gold electrode of the TSR and the hydrogel.

- Polymer film formation through spin-coating of an aqueous solution of PVA (PolySciences, $M W \approx 125,000,88$ mol. \% hydrol.) and PAA (PolySciences, $M W \approx 450,000$ ) in the ratio 8:1.

- Thermal cross-linking of the two polymers through the formation of ester bonds between the hydroxyl groups and the carboxylic acid group. 
The thickness $d$ and homogeneity $s^{2}(d)$ of the hydrogel film are predefined through both the interrelation of the polymer solution concentration and the speed profile during the spin-coating. In the subsequent thermal cross-linking step, the temperature profile determines the complex shear modulus $G^{*}$ and the range of its concentration-dependent changes. This allows the separate optimization for both parameters without disturbing interrelations.

Fig. 2 shows the process oriented approach for the application specific sensor optimization. The goal is defined by the application, which requires certain sensor properties in order to fulfil the measurement task. The measurement model matches the characteristic curve to the requirements within certain limits, provided that the coated TSR has a sufficient sensitivity ${ }^{1}$.
Considering these interdependencies, the goal of the process optimization is to find the value ranges of the process parameters that ensure the desired film properties and consequently a sufficient sensitivity. In a first step, this requires a detailed understanding of both the interrelations between the hydrogel film properties and the resulting behavior of the coated TSR. From this, target values for $d, G$ ' and G' can be defined and used as quality monitoring features. The second step is then an experimental investigation of the interrelation between the process parameters and these monitoring features. Please note that this approach for the process optimization requires measurement procedures for the thickness and the mechanical properties of thin hydrogel films.

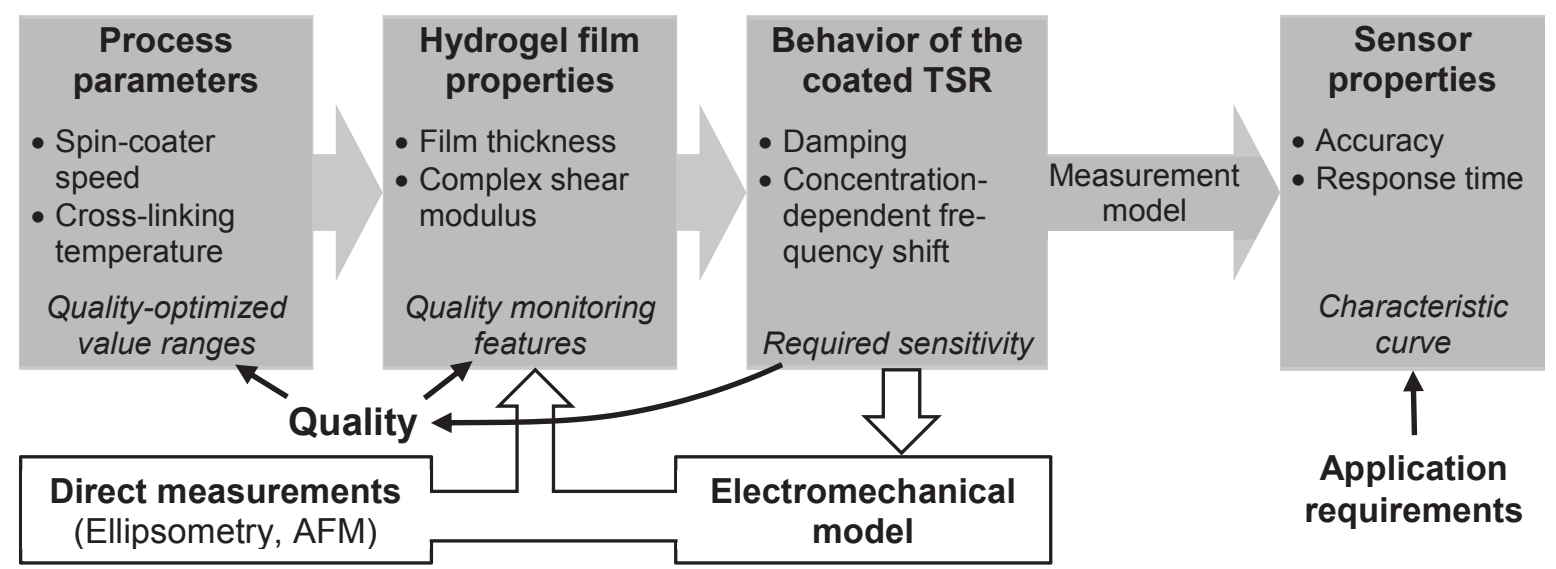

Fig. 2: Interrelations and optimization of the manufacturing process

\section{Measurement Procedures for Hydrogel Films Properties}

Direct measurements are the first option for investigating the properties of visco-elastic hydrogel films in the submicrometer range. Amongst various tested procedures ${ }^{2}$ for measuring the thickness of dry and swollen hydrogel films, spectral ellipsometry and atomic force microscopy (AFM) provided the best results. A variable angle spectroscopic ellipsometer (VASE, M2000 by J.A. Woollam Co.) was used for measuring the thickness $d$ and refractive index $n$ of hydrogel layers on TSRs simultaneously. This additional information on the optical hydrogel properties can be used to evaluate its chemical homogeneity and to some extend its material density.

\footnotetext{
${ }^{1}$ A sufficient selectivity is a second important property. Since it solely depends on the hydrogel type, it is not taken into account in this context.
}

Thickness measurements with AFM require a sharp step between the substrate and the film surface, which can be generated by scratching the hydrogel. It allows reliable direct measurements of the film thickness. In addition, AFM can simultaneously measure the stiffnes $G$ ' (Young's modulus) of the hydrogel. However, the effective glass transition temperature of polymers, and therefore G' and G', strongly depends on the measurement frequency [5]. Since the cantilever frequency of AFM is usually in the dimension of $10^{1} \ldots 10^{2} \mathrm{kHz}$, while the TSR works at $10 \mathrm{Mhz}$, the effective values of $G^{*}$ differ. A conversion is not possible, because the necessary interrelations, respectively the constants of the WLF-equation [5], are still unknown for the specific PVA/PAA hydrogel. Consequently, the directly measured material

\footnotetext{
2 The following tested measurement procedures were found to be not applicable for different reasons: reflectometric interference spectroscopy (RIfS), X-ray reflectometry (XRR), confocal microscopy ( $\mu$ Scan).
} 
properties can currently only be used for relative comparisons.

A second option for investigating the hydrogel film properties are indirect measurements using the coated TSR itself as quartz crystal microbalance (QCM). In this approach, an analytical electromechanical model of the quartz crystal and the attached visco-elastic layer is used to simulate its behavior in the frequency domain. By matching the simulated impedance spectra to the measured ones, the corresponding values for $d$ and $G^{*}$ can be extracted from the model [4], [6]. This modelling approach has provided beneficial qualitative insights in the interrelations between the film properties and the sensor behavior. Its validation for sufficiently precise quantitative analyses is subject to current research activities.

\section{Spin-Coating of Homogenous Polymer Films}

Research objectives for the optimization of the spin-coating step are (1) the creation of uniform hydrogel films with a reproducible thickness within the batch and (2) the development of a mathematical expression for the interrelations between the process parameters and the resulting thickness. For acquiring the necessary experimental data, 16 TSRs were coated using different polymer concentrations and rotation speeds ${ }^{3}$ according to table 1 . Subsequently, the film thickness was measured with the M-2000 ellipsometer in dry state. In addition, the viscosity of the polymer solutions was measured at room temperature for different shear rates (Anton Paar Pysica MCR 301; Cone-Plate $\varnothing 50$ mm, angle $1^{\circ}$ ).

From the ellipsometry results the following statistical parameters were calculated:

- $\bar{d}$ : average thickness within one film

- $X(\bar{d})$ : average of $\bar{d}$ within the batch

- $s^{2}(\bar{d})$ : variance of the average film thickness within the batch

- $s^{2}(d)$ : thickness variance within one film

- $X\left[s^{2}(d)\right]$ : average of these variances within the batch

The values of these parameters in table 1 illustrate the good homogeneity and reproducibility of the films. In agreement with qualitative simulation results of the electromechanical model can be concluded, that such small deviations of the

\footnotetext{
3 The polymer solution was preconditioned and equally spread on the TSRs by rotating at 250 rpm for 30 s. Subsequently, the spin-coater
}

thickness are not the reason for the observed variance of the sensor sensitivity.

The second objective of the experimental investigation of the spin-coating step was the development of an equation for precalculating the film thickness from the process parameters. Based on the works of Meyerhofer [7] and Spangler et. al. [8], eq. (1) was chosen as general relation for spin-coating.

$$
d=k \frac{c_{P}^{x}}{\omega^{y}}
$$

Deviating from the mentioned publications, the polymer concentration $C_{P}$ is used as parameter instead of the initial ${ }^{4}$ solution viscosity $\eta_{0}$, based on the approximation given in eq. (2) [8].

$$
\begin{gathered}
\eta_{0}=\eta_{\text {Solvent }}+\eta_{\text {Solid }} c^{x} \approx k c^{x} \\
\text { for } \eta_{\text {Solvent }} \ll \eta_{\text {Solid }} c^{x}
\end{gathered}
$$

The motivation for this approximation is the pronounced shear-thinning behavior of the polymer solution (tab. 1). Its viscosity decreases with increasing shear rates $s$. Since $s$ is unknown and not constant in the polymer film during the spincoating, it is ambiguous at which shear rate the value $\eta_{0}$ is to be measured. Therefore $c_{P}$ is used as more feasible parameter.

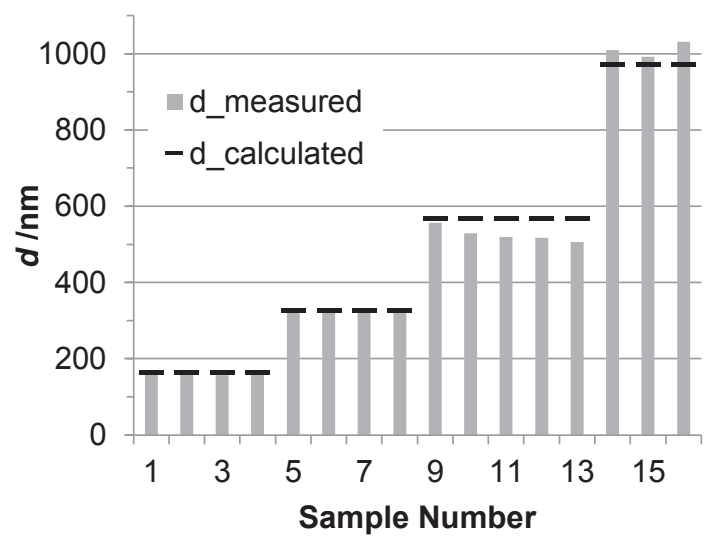

Fig. 3: Measured and calculated film thickness

The proportionality factor $k$ is a polymer specific constant which needs to be determined for PVA/PAA solution. Furthermore, different values had been reported for the exponents $x$ and $y$. This implies that they depend on the polymersolvent-system, too. Consequently, all three parameters were determined from the experimental results by minimizing the least mean square error between the calculated and the measured thickness. With the resulting values $k=1600 \mathrm{~nm}^{*} \mathrm{~min}^{-1 / 2}, x=1.9940$ and $y=0.4998$ the average relative error was $3.8 \%$ (Fig. 3 ).

accelerated to the denoted $\omega$ and remained at that speed until the films were dry.

4 "Initial“ means before solvent is vaporizing during the spin-coating, which increases $\eta$. 
Hence, the proposed equation describes the interrelation between the process parameters and the hydrogel film properties for the spin-coating step sufficiently well. Remarkable is also the excellent conformity with Meyerhofer's experimental results $(x=2$ and $y=0.5)$, although the shear-thinning phenomenon had not been taken into account for the underlying theoretical considerations.

Tab. 1: Ellipsometry measurements of PVA/PAA films with different coating parameters

\begin{tabular}{|c|c|c|c|c|c|c|c|c|c|c|}
\hline \multirow{2}{*}{$\begin{array}{c}\text { Batch- } \\
\text { No. }\end{array}$} & \multirow{2}{*}{$\begin{array}{c}\text { No. of } \\
\text { TSRs }\end{array}$} & \multirow{2}{*}{$\begin{array}{c}c_{p} \\
\text { /wt. } \%\end{array}$} & \multirow{2}{*}{$\begin{array}{c}\omega \\
/ \mathrm{min}^{-1}\end{array}$} & \multicolumn{2}{|c|}{$\eta /$ Pa.s for different shear rates $s / \mathrm{s}^{-1}$} & & $X(\bar{d}) /$ & \multirow{2}{*}{$\begin{array}{c}s^{2}(\bar{d}) \\
/ \mathrm{nm}\end{array}$} & $\begin{array}{c}X\left[s^{2}(d)\right] \\
/ \mathrm{nm}\end{array}$ \\
\hline 1 & 4 & 2.5 & 3900 & 0.0823 & 0.0778 & 0.0620 & 0.0404 & 159.3 & 1.7 & 6.9 \\
\hline 2 & 4 & 3.5 & 3750 & 0.2332 & 0.2028 & 0.1530 & 0.0949 & 323.4 & 2.6 & 5.8 \\
\hline 3 & 5 & 4.5 & 3350 & 0.4920 & 0.4253 & 0.3008 & 0.1773 & 525.5 & 17.0 & 7.9 \\
\hline 4 & 3 & 5.5 & 2500 & 1.3960 & 1.0842 & 0.7155 & 0.4119 & 1011.2 & 16.2 & 7.0 \\
\hline
\end{tabular}

\section{Thermal Cross-linking}

A second batch of 13 TSRs was spin-coated with equal parameters $\left(C_{P}=2.5 \mathrm{wt}\right.$. $\left.\% ; \omega=2500 \mathrm{~min}^{-1}\right)$ and evenly distributed in a laboratory oven (Binder VD 23) for the thermal cross-linking at $160^{\circ} \mathrm{C}$ for $20 \mathrm{~min}$. The subsequent measurements showed large sensitivity differences of the sensors towards the selected industrial cleaner (sodium pyrophosphate). These differences could be correlated to the position in the oven (Fig. 5). Furthermore, the Young's modulus $E$ of five selected films was measured in swollen state with the AFM at 75 different points on the surface. Hydrogels with a high sensitivity had a Young's modulus in the range of $45 \mathrm{kPa}$; for the ones with a low sensitivity $E$ was around $25 \mathrm{kPa}$. Considering the direct interrelation between the Young's modulus and the cross-linking degree, this indicates a non-uniform cross-linking of the hydrogel layers in the oven [9]. The strong nonlinear interrelation between temperature, time and resulting cross-linking degree for PVA/PAA [10] requires a precise temperature profile.

Measurements of the temperature field after inserting the coated TSRs into the preheated oven show a very inhomogeneous temperature distribution with local differences of up to $10 \mathrm{~K}$ (Fig. 4). The open door seems to generate an air swirl which cools the outer parts and leaves a hot spot in the middle during the insertion time (about $30 \mathrm{sec}$ ).

The similarities between the spatial distribution of temperature and sensitivity in the figures 5 \& 6 suggest the following explanation for the observed sensitivity deviations: The hot spot leads to a higher cross-linking degree of the hydrogel films in the middle of the oven. A consequence of that is a higher Young's modulus of these hy- drogels, which corresponds to a higher sensitivity, as the AFM measurements indicate. A possible reason, why higher cross-linked and thus less swellable hydrogels exhibit a better sensitivity, relates to the transducer principle: Too soft hydrogels do not sufficiently follow the shear oscillation of the TSR and possess therefore less influence on the vibration behavior of the system, even if they strongly swell.

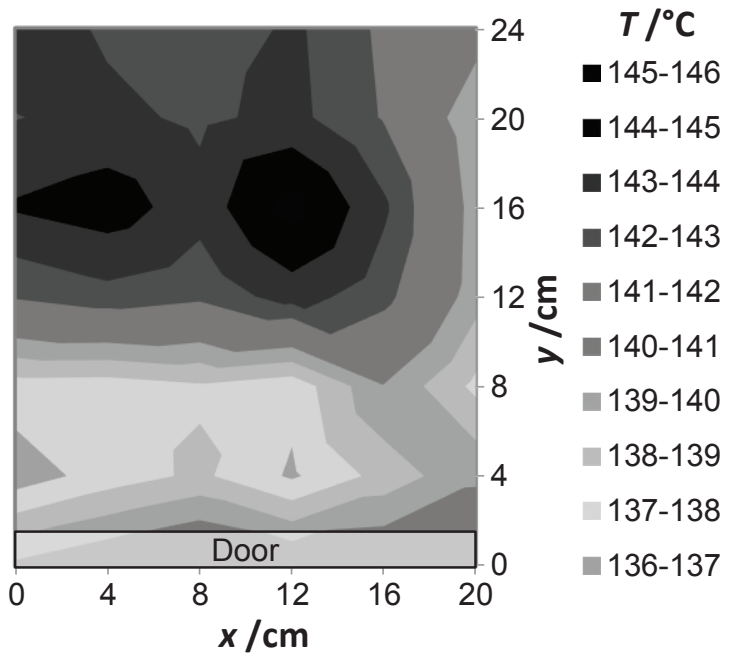

Fig. 4: Temperature distribution in cross-linking oven after inserting the coated quartz crystals

A batch manufacturing tool made of massive copper for up to 24 TSRs was designed in order to unify and control the cross-linking temperature. It reduces the spatial temperature differences to less than $0.3 \mathrm{~K}$ and has an integrated sensor for real time temperature monitoring. In first tests this allowed the reproducible manufacturing of sensors with a significantly increased sensitivity of up to $1500 \mathrm{~Hz} / \mathrm{vol} .-\%$.

In the future, an experimental series with different temperatures and cross-linking times is planned. The aim is to develop a mathematical 
description for the interrelations between process parameters and hydrogel film properties for the cross-linking step, similar to the one which

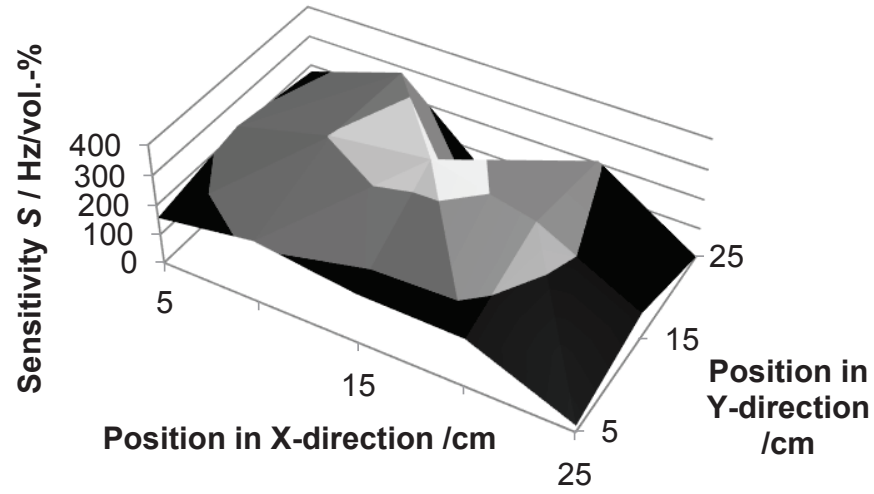

has been successfully developed for the spincoating.
Good sensitivity $\rightarrow$ high accuracy

$200 \mathrm{~Hz} / \% \leq \mathrm{S} \leq 300 \mathrm{~Hz} / \%$ :

Small sensitivity $\rightarrow$ reduced accuracy

$\mathbf{S}<200 \mathrm{~Hz} / \%$ :

Insufficient sensitivity $\rightarrow$ waste

Fig. 5: Effect of the inhomogeneous cross-linking temperature distribution in the oven on the resulting sensitivity and, consequently, the functionality of the sensor

\section{Conclusions}

A methodic approach for the optimization of thin hydrogel films was successfully applied to an industrial hydrogel sensor for surface technology. The proposed process is easily scalable and well controllable because of the separation of the film formation and the cross-linking step. Due to these advantages, we suggest to use it as manufacturing standard and adapt the hydrogel synthesis, if necessary (and not vice versa). For example, Hirata et. al. [11] showed that a previously only chemically cross-linkable hydrogel can be thermally cross-linked as well.

Future research will cover the validation of the electromechanical model for indirect measurements of hydrogel film properties and the development of a mathematical description of the cross-linking step. The general aim is to provide a well investigated, versatile and controllable manufacturing process for the product development of commercially available hydrogel sensors.

\section{Acknowledgements}

This research was funded by a PhD scholarship of the German National Academic Foundation. Previous financial support of the R\&D project "Prozessmesstechnik zur Badueberwachung in der Oberflaechentechnik mittels Hydrogelsensoren" by the EU (ERDF, European Regional Development Funds) and the Free State of Saxony is gratefully acknowledged. The authors also thank the former project partner SITA Messtechnik $\mathrm{GmbH}$ for the supplied material and S. Abril-Guevara, A. Gehre, L. Guenther, J. Nowak and $\mathrm{Y}$. Wang for supporting the experiments.

\section{References}

[1] I. Tokarev, S. Minko, Soft Matter 5, 511-524 (2009); doi: 10.1039/B813827C
[2] M. Windisch, T. Junghans, Hydrogel Sensors for Process Monitoring, Adv. in Science and Technology 77, 71.76 (2013); doi:10.4028/www.scientific.net/AST.77.71

[3] M. Windisch, T. Junghans, Innovative Hydrogel Sensor Solution for Process Monitoring, ScieConf 2014, Zilina (Slovakia), 09.-13.06.2014; ISBN: 978-80-554-0891-0

[4] R. Bruenig, Modellierung von akustischen Dickenscherschwingern im Frequenzbereich, Dissertation (2011)

[5] M. L. Williams, R. F. Landel, J. D. Ferry, The temperature dependence of relaxation mechanisms in amorphous polymers and other glass forming liquids, J. Am. Chem. Soc. 77 3701-7 (1955)

[6] C. Behling, R. Lucklum, P. Hauptmann, Response of quartz-crystal resonators to gas and liquid analyte exposure, Sensors and Actuators A 68, 388-398 (1998); doi: 10.1016/S09244247(98)00088-0

[7] D. Meyerhofer, Characteristics of resist films produced by spinning, Journal of Applied Physics 49, 3993 (1978); doi:10.1063/1.325357

[8] L. L. Spangler, J. M. Torkelson, J. S. Royal, Influence of Solvent and Molecular Weight in Thickness and Surface Topography of Spin-Coated Polymer Films, Polymer Engineering and Science, Vol. 30, No. II (1990)

[9] G. Gerlach, K.-F. Arndt, Hydrogel Sensors and Actuators, Springer-Verlag (2009); doi: 10.1007/978-3-540-75645-3

[10] K.-F. Arndt et. al., Poly(vinyl alcohol)/poly(acrylic acid) hydrogels: FT-IR spectroscopic characterization of crosslinking reaction and work at transition point, Acta Polymerica 50, 383-390 (1999)

[11] I. Hirata, M. Okazaki, H. Iwata, Simple method for preparation of ultra-thin poly(n-isopropylacrylamide) hydrogel layers and characterization of their thermo-responsive properties, Polymer 45, 5569-5578 (2004); doi: 10.1016/j.polymer.2004.06.015 